\title{
EFECTIVIDAD DEL TRATAMIENTO DEL DOLOR IRRUPTIVO ONCOLÓGICO CON COMPRIMIDO BUCAL DE FENTANILO (CBF) REVISIÓN BIBLIOGRÁFICA DE SU FARMACOCINETICA, SEGURIDAD Y EMPLEO EN SITUACIONES CLÍNICAS ESPECIALES
}

\author{
EFFECTIVENESS OF FENTANYL BUCCAL TABLET FOR THE TREATMENT OF \\ BREAKTHROUGH CANCER PAIN. REVIEW ON PHARMACOKINETICS, SAFETY AND \\ CLINICAL USE IN SPECIAL SITUATIONS
}

Maite Murillo González', Dulce Rodríguez Mesa², Yolanda Escobar Álvarez ${ }^{3}$ y Jordi Moya Riera ${ }^{4}$

1 Servicio de Oncología Radioterápica, Hospital Universitario 12 de Octubre, Madrid.

2 Unidad de Cuidados Paliativos, Hospital Sant Joan de Reus, Tarragona.

${ }^{3}$ Servicio de Oncología Médica. Hospital General Universitario Gregorio Marañón, Madrid.

${ }_{4}^{4}$ Unidad del Dolor, Hospital Mateu Orfila, Menorca.

\section{Resumen}

Varios estudios han mostrado que la prevalencia del dolor irruptivo en pacientes oncológicos es elevada y que se trata de un problema no completamente resuelto. El tratamiento del dolor irruptivo oncológico (DIO) requiere un fármaco con características específicas siendo probablemente de elección fentanilo, que se presenta en diversas formulaciones. En este trabajo se ha realizado una revisión de los ensayos clínicos publicados sobre el comprimido bucal de fentanilo para valorar la evidencia disponible en relación a su efectividad en el tratamiento del dolor irruptivo oncológico y aspectos relacionados con su farmacocinética, seguridad y particularmente su empleo en condiciones especiales como la mucositis. La evidencia revisada muestra que el comprimido bucal de fentanilo es eficaz en términos de alivio del dolor en el tratamiento de los episodios de DIO, incluyendo los pacientes con mucositis. Se ha revisado también la bioequivalencia entre la administración bucal (entre la encía y la mejilla) y sublingual (bajo la lengua) del preparado.
Abstract

Several studies have shown that the prevalence of breakthrough pain in cancer patients is high and it is a problem not fully solved. Treatment of breakthrough cancer pain requires a drug with specific characteristics and probably the most useful drug is fentanyl, that is available in several formulations. This paper presents a review of published clinical trials on fentanyl buccal tablet to assess the evidence available regarding its effectiveness in the treatment of breakthrough cancer pain, issues related to its pharmacokinetics, safety and its use in special conditions such as mucositis. Evidence shows that fentanyl buccal tablet is effective for relieving pain in the treatment of episodes in breakthrough pain, including patients with mucositis. The bioequivalence of buccal (between the gum and cheek) and sublingual (under the tongue) administration has been also reviewed.

Key words: Breakthrough cancer pain, safety, effectiveness, fentanyl buccal tablet.

Palabras clave: Dolor irruptivo oncológico, seguridad, efectividad, comprimido bucal de fentanilo. 


\section{INTRODUCCIÓN}

Se define el dolor irruptivo como una exacerbación transitoria del dolor que se produce en un paciente que tiene un dolor de base controlado en relación con un desencadenante conocido o no ${ }^{(1)}$. Cuando la causa del dolor de base y los episodios de dolor irruptivo tienen de origen una patología oncológica se define como dolor irruptivo oncológico (DIO) y se caracteriza por mostrar un inicio rápido, alcanzando con frecuencia una intensidad máxima en un plazo de minutos ${ }^{(1)}$. Varios estudios han mostrado que la prevalencia del dolor irruptivo en pacientes oncológicos con dolor persistente pero controlado, oscila entre el $51 \%$ y el $89 \%{ }^{(1,2)}$.

En el tratamiento del DIO se emplean diversas intervenciones, con resultados variables ${ }^{(1)}$. En este trabajo se ha realizado una revisión de los artículos publicados sobre el comprimido bucal de fentanilo (CBF) para valorar la evidencia disponible en relación a su efectividad en el tratamiento del dolor irruptivo oncológico y aspectos relacionados con su farmacocinética, seguridad y empleo en condiciones especiales.

\section{MÉTODOS}

Se propone identificar los estudios sobre el CBF en las siguientes fuentes de información: MEDLINE, EMBASE y Cochrane Database of Systematic Reviews. Se resumirán los resultados para las diferentes valoraciones de interés así como los registros de estimación del efecto disponibles y las variables de resultados. Se han incluido los estudios observacionales y los ensayos clínicos controlados excluyéndose los artículos de revisión y similares.

Se identificaron 22 ensayos, de los que se seleccionaron $9^{(3-11)}$ y se excluyeron $13^{(12-24)}$ por no referirse a las condiciones indicadas, es decir dolor exclusivamente oncológico, CBF en estudio de eficacia, farmacocinética, seguridad o su empleo en condiciones especiales. También se excluyeron duplicidades, revisiones o trabajos de valoración sobre estudios publicados. Recientemente se ha realizado un estudio fase IV a nivel europeo sobre el CBF con 330 pacientes, cuyos resultados no están disponibles en su totalidad, aunque se han publicado algunos aspectos relacionados con metodología, titulación de la dosis y calidad de vida ${ }^{(25,26)}$.

\section{Resultados: eficacia del CBF en el tratamiento del DIO}

En un ensayo clínico fase III, Portenoy et al. (3) estudiaron 77 pacientes con DIO tratados con una pauta fija $\geq 60 \mathrm{mg} /$ día de morfina o 50-300 $\mu \mathrm{g}$ de fentanilo transdérmico que presentaban episodios de DIO. Previamente se realizó un ajuste de dosis para determinar la dosis eficaz de fentanilo. En estos pacientes 493 episodios de dolor irruptivo fueron tratados con CBF y 208 con placebo. EI SPID (sumatorio de la diferencia en la intensidad del dolor) a los 30 minutos de la administración del CBF o placebo fue la variable principal del estudio, los resultados se exponen en la tabla 1.

Slatkin et al. ${ }^{(4)}$ en otro ensayo también fase III, evaluaron como resultado principal el SPID 60 : sumatorio de todas las diferencias de intensidad del dolor desde los 5 hasta los 60 minutos. Se evaluaron 78 pacientes y tras una fase de ajuste de dosis, los pacientes fueron asignados aleatoriamente para tratar 10 episodios de $\mathrm{DIO}$ con 10 comprimidos ( 7 con CBF, 3 con placebo). Los resultados se exponen en la tabla 1. La diferencia en la proporción de episodios de DIO con una mejoría del PI (Intensidad del Dolor) $\geq 50 \%$ fue estadísticamente significativa a los 10 minutos a favor del CBF comparado con placebo.

Los resultados de ambos estudios in- 


\section{Tabla 1. CBF versus placebo. Sumatoria de la diferencia en la intensidad del dolor.}

\begin{tabular}{|l|c|c|c|c|}
\cline { 2 - 5 } \multicolumn{1}{c|}{} & Variable & CBF Media & Placebo media & CBF versus placebo \\
\hline Portenoy et al. $(2006)^{3}$ & SPID $_{30}$ & $3( \pm 0,12)$ & $1,8( \pm 0,18)$ & $\mathrm{p}<0,0001$ \\
\hline Slatkin et al. $(2007)^{4}$ & SPID $_{60}$ & $9,7( \pm 0,63)$ & $4,9( \pm 0,5)$ & $\mathrm{p}<0,0001$ \\
\hline
\end{tabular}

CBF: comprimido bucal de fentanilo; SPID: sumatoria de la diferencia en la intensidad del dolor.

dican que con el CBF, la probabilidad de obtener un efecto analgésico estadísticamente significativo y clínicamente importante (definido de las diversas formas antes señaladas) fue mayor (más del doble) que con placebo (OR entre 2,39 y 2,63).

\section{Valoración del riesgo de sesgo}

El mayor detalle en la descripción de los aspectos metodológicos del estudio de Slatkin et al. ${ }^{(4)}$ permite calificar este estudio como de alta calidad, con un bajo riesgo de sesgo.

Con el objetivo de evaluar la consistencia y relevancia clínica de los resultados de eficacia, en concordancia con el reciente consenso del grupo IMMPACT, Zeppetella et al.(5) realizaron un análisis combinado de ambos ensayos clínicos. Observaron que la proporción de episodios de DI con una mejora en la puntuación de la intensidad del dolor $\geq 33 \%$ (moderado) o $\geq 50 \%$ (importante) respecto al valor basal, fue significativamente mayor en los pacientes tratados con CBF en comparación con placebo, en todos los puntos temporales $(p<0,0001)$.

Resultados: velocidad de absorción y aspectos farmacocinéticos relacionados

Pather et al. ${ }^{(6)}$ estudiaron la farmacocinética de la dosis de $200 \mu$ g de fentanilo administrados en tres preparaciones diferentes: comprimido bucal efervescente $\left(\mathrm{CBF}\right.$, OraVescent $\left.{ }^{\circledR}\right)$, comprimido bucal no efervescente y citrato de fentanilo oral transmucoso (CFOT). Los niveles de fentanilo en plasma fueron determinados cada 2 minutos durante los primeros 6 minutos y después cada 3 minutos en los 15 minutos siguientes. Los resultados se exponen en la tabla 2.

Darwish et al. ${ }^{(7)}$ realizaron un ensayo aleatorizado en 26 sujetos sanos. Cada participante fue tratado con $400 \mu \mathrm{g}$ de CBF (transmucoso), $800 \mu \mathrm{g}$ de CBF (oral), $800 \mu \mathrm{g}$ de CFOT y $400 \mu \mathrm{g}$ de fentanilo intravenoso. $\mathrm{La} \mathrm{T}_{\max }$ fue más corta con CBF transmucoso que con CBF oral o CFOT. También se observó un valor más alto de $\mathrm{C}_{\max }$ y una mayor exposición sistémica con la preparación de CBF transmucoso (tablas 3 y 4).

Para determinar la proporcionalidad de la dosis de CBF, Darwish et al. ${ }^{(8)}$ realizaron un estudio aleatorizado y abierto en 120 sujetos sanos. Se administraron secuencias aleatorias de 600, 1.000, 1.200 y 1.300 $\mu \mathrm{g}$ del fármaco. Se observaron relaciones aproximadamente lineales entre la dosis y los parámetros farmacocinéticos dependientes de la dosis $\mathrm{C}_{\max }$ y $A \cup \mathrm{C}_{\infty}$ por lo que es esperable un incremento lineal en la exposición sistémica entre las dosis indicadas.

Resultados: administración sublingual del CBF

El CBF está formulado para mejorar su 
Tabla 2. Pather et al.(6) registraron una absorción más rápida ( $\mathbf{T}_{\max }$ más corta) y más completa (mayor AUC) y niveles séricos mayores con FTB. Estas diferencias fueron estadísticamente significativas.

\begin{tabular}{|c|c|c|c|}
\cline { 2 - 4 } \multicolumn{1}{c|}{} & $\begin{array}{c}\text { Fentanilo OraVescent } \\
\text { CBF }\end{array}$ & $\begin{array}{c}\text { Fentanilo bucal no } \\
\text { efervescente }\end{array}$ & $\begin{array}{c}\text { Citrato de fentanilo } \\
\text { oral transmucoso. }\end{array}$ \\
\hline $\mathrm{C}_{\max }(\mathrm{ng} / \mathrm{mL})$ & $0,6412( \pm 0,2804)$ & $0,39886( \pm 0,0744)$ & $0,4073( \pm 0,1537))$ \\
\hline $\mathrm{A} \cup \mathrm{C}(\mathrm{Ot})$ & $2.656( \pm 0,6729)$ & $2,041( \pm 0,8690)$ & $1,809( \pm 0,9358)$ \\
\hline $\mathrm{T}_{\text {max }}$ (horas) & 0,501 & 2 & 2 \\
\hline
\end{tabular}

AUC: área bajo la curva. Indica la concentración o absorción de un medicamento en función de un período de tiempo. Cmax: concentración plasmática máxima. Tmax: tiempo necesario para alcanzar la concentración máxima de un fármaco.

Tabla 3. Biodisponiilidad absoluta de fentanilo. AUC $_{0-\infty}$ : Área bajo la curva de la concentración plasmática desde el tiempo 0 a $\infty$. Ratio individual: Ratio de la dosis normalizada de $\mathrm{AUC}_{0-\infty}$ para cada formulación de fentanilo. Modificada de Darwish et al.(7)

\begin{tabular}{|c|c|c|c|c|}
\cline { 2 - 5 } \multicolumn{1}{c|}{} & $\mathrm{CBF}_{\text {trm }} 400 \mu \mathrm{g}$ & $\mathrm{CBF}_{\text {oral }} 800 \mu \mathrm{g}$ & $\mathrm{CFOT}_{\text {trm }} 800 \mu \mathrm{g}$ & Fentanilo $_{\text {iv }} 400 \mu \mathrm{g}$ \\
\hline $\mathrm{AUC}_{0-\infty \text { (media } \pm \mathrm{DE})}$ & $6,48 \pm 2,98$ & $6,60 \pm 4,47$ & $9,58 \pm 3,91$ & $10,29 \pm 2,88$ \\
\hline Ratio individual & $\begin{array}{c}0,65 \\
(0,51-0,70)\end{array}$ & $\begin{array}{c}0,31 \\
(0,24-0,33)\end{array}$ & $\begin{array}{c}0,47 \\
(0,40-0,54)\end{array}$ & -- \\
\hline
\end{tabular}

AUC: área bajo la curva. Indica la concentración o absorción de un medicamento en función de un período de tiempo. CBF: comprimido bucal de fentanilo. CFOT: citrato de fentanilo oral transmucoso.

Tabla 4. Exposición temprana a fentanilo medida a través de $\mathbf{C}_{\max } \mathbf{Y} \mathbf{A U C}_{0-\operatorname{tmax}} \mathbf{Y}$ exposición sistémica total medida a través de $\mathrm{AUC}_{0-\infty}$. Todos los valores están expresados en media $\pm D E$. Modificado de Darwish et al. ${ }^{(7)}$

\begin{tabular}{|c|c|c|}
\cline { 2 - 3 } \multicolumn{1}{c|}{} & $\mathrm{CBF}_{\text {trm }}$ & CFOT $_{\text {trm }}$ \\
\hline $\mathrm{C}_{\text {max }} \mathrm{ng} / \mathrm{mL}$ & $1,02 \pm 0,42$ & $0,63 \pm 0,21$ \\
\hline $\mathrm{A} \cup \mathrm{C}_{0-\infty} \mathrm{ng} \cdot \mathrm{h} / \mathrm{mL}$ & $6,48 \pm 2,98$ & $4,79 \pm 1,96$ \\
\hline $\mathrm{A} \cup \mathrm{C}_{0-\text { tmax }} \mathrm{ng} \cdot \mathrm{h} / \mathrm{mL}$ & $0,40 \pm 0,18$ & $0,14 \pm 0,05$ \\
\hline
\end{tabular}

AUC: área bajo la curva. Indica la concentración o absorción de un medicamento en función de un período de tiempo. Cmax: concentración plasmática máxima. CBF: comprimido bucal de fentanilo. CFOT: citrato de fentanilo oral transmucoso. IC: Intervalo de confianza. OD: odds ratio. PI: intensidad del dolor. Tmax: tiempo necesario para alcanzar la concentración máxima de un fármaco. 
absorción a través de la mucosa bucal, Darwish $^{(9)}$ estudió la bioequivalencia entre una dosis bucal de $400 \mu \mathrm{g}$ (entre la encía y la mejilla) y sublingual (colocada bajo la lengua). Participaron 90 sujetos sanos de los cuales completaron el estudio 78 y en los que la ratio bucal/sublingual de la medias de la Cmax y el AUC $\infty$ se mostró dentro del rango de bioequivalencia. Ratio sublingual/bucal para Cmax $=0,868(90 \%$ $\mathrm{Cl} 0,815,0,924)$; ratio sublingual/bucal para $A \cup C \infty=0,947(90 \% \mathrm{Cl} 0,901,0,995)$.

Los resultados apoyaron el uso del CBF sublingual como alternativa viable en pacientes que requieran una administración alternativa a la bucal por condiciones de salud bucodental, necesidad de un cuidador para la administración o mucositis ${ }^{(9)}$.

Resultados de la administración en condiciones especiales: presencia de mucositis oral

Darwish et al. ${ }^{(10)}$ estudiaron la administración del CBF en pacientes oncológicos con mucositis oral. En un estudio abierto se determinaron los parámetros farmacocinéticos y los acontecimientos adversos tras la administración de $200 \mu \mathrm{g}$ del CBF en pacientes con y sin mucositis. Los pacientes presentaban grados de mucositis clínica entre 1-3 y funcional entre 1-2. No se dispone de datos en pacientes con grados de mucositis más severos.

El perfil de absorción tras esta dosis de fentanilo fue similar en los dos grupos y no se observaron diferencias significativas entre los pacientes con y sin mucositis en los valores de las medias de Cmax y la Tmax. Tampoco se registraron diferencias significativas en cuanto a efectos adversos.

\section{RESULTADOS}

\section{Seguridad y tolerabilidad}

Weinstein et al. ${ }^{(11)}$ realizaron un estudio de seguridad a largo plazo en 232 pacientes. Aproximadamente la mitad había participado en ensayos previos, en los cuales se había identificado la dosis eficaz del CBF. En el resto se procedió previamente a un ajuste de dosis para determinar la dosis eficaz. Se considera aconsejable monitorizar al paciente durante el proceso de ajuste de dosis y tener en cuenta el riesgo potencial de depresión respiratoria. Los pacientes fueron estudiados durante 12 meses.

En general, el tratamiento con CBF durante $\geq 12$ meses fue bien tolerado en pacientes con dolor irruptivo oncológico. La seguridad y tolerabilidad fue similar a la observada en los estudios a corto plazo. Los efectos adversos notificados con más frecuencia se indican en la tabla 5.

La mayoría de los pacientes valoraron el tratamiento con CBF como bueno o muy bueno, medido a través del cuestionario Global Medication Perfomance. La puntuación fue relativamente estable en los pacientes que permanecieron en el estudio hasta el final(11).

Dos ensayos clínicos controlados han valorado la seguridad del CBF a corto plazo $^{(3-4)}$. Los pacientes incluidos ya estaban siendo tratados con opiodes para el control del dolor y por lo tanto habían desarrollado un cierto grado de tolerancia a los efectos adversos de los opioides. La mayoría de los acontecimientos adversos fueron leves o moderados y los graves se relacionaron principalmente con la enfermedad de base. En la tabla 6 se exponen los acontecimientos adversos notificados con mayor frecuencia.

Entre los aspectos de seguridad puede incluirse también la dosificación inicial en la titulación de la dosis eficaz. En un estudio de no inferioridad, paneuropeo, multicéntrico, en fase IV sobre 320 pacientes con DOI, Davies et al. ${ }^{(25)}$ estudiaron el porcentaje de pacientes que alcanzaron la dosis de analgesia adecuada y sin aconte- 
Tabla 5. Efectos adversos notificados con mayor frecuencia en el estudio de Weinstein et al.(11)

\begin{tabular}{|c|c|c|c|}
\cline { 2 - 4 } \multicolumn{1}{c|}{} & Total & $\begin{array}{c}\text { Weinstein } \\
\text { Fase de titulación } \\
(\mathrm{n}=112)\end{array}$ & $\begin{array}{c}\text { Weinstein. } \\
\text { Fase de tratamiento } \\
(\mathrm{n}=197)\end{array}$ \\
\hline Náuseas & $37 \%$ & $24 \%$ & $32 \%$ \\
\hline Mareo & $20 \%$ & $26 \%$ & $11 \%$ \\
\hline Fatiga & $16 \%$ & $3 \%$ & $18 \%$ \\
\hline Vómitos & $22 \%$ & $4 \%$ & $24 \%$ \\
\hline Estreñimiento & $14 \%$ & $3 \%$ & $15 \%$ \\
\hline Anemia & $14 \%$ & $3 \%$ & $15 \%$ \\
\hline Cefalea & $14 \%$ & $10 \%$ & $12 \%$ \\
\hline Somnolencia & $13 \%$ & $13 \%$ & $9 \%$ \\
\hline Edema & $13 \%$ & $0 \%$ & $15 \%$ \\
\hline Dolor abdominal & $11 \%$ & $2 \%$ & $12 \%$ \\
\hline
\end{tabular}

Tabla 6. Efectos adversos notificados con mayor frecuencia en los estudios de Slatkin et al. $^{(4)}$ y Portenoy et al. $^{(3)}$

\begin{tabular}{|c|c|c|}
\cline { 2 - 3 } \multicolumn{1}{c|}{} & $\begin{array}{c}\text { Slatkin et al. } \\
(\mathrm{n}=125)\end{array}$ & $\begin{array}{c}\text { Portenoy et al. }{ }^{(3)} \\
(\mathrm{n}=123)\end{array}$ \\
\hline Náuseas & $13 \%$ & $22 \%$ \\
\hline Mareos & $11 \%$ & $22 \%$ \\
\hline Fatiga & $8 \%$ & $12 \%$ \\
\hline Vómitos & $6 \%$ & $11 \%$ \\
\hline Cefalea & $6 \%$ & $15 \%$ \\
\hline Estreñimiento & $6 \%$ & $8 \%$ \\
\hline Somnolencia & - & $10 \%$ \\
\hline Astenia & - & $7 \%$ \\
\hline
\end{tabular}

Tabla 7. Porcentaje de pacientes que alcanzaron la dosis de respuesta analgésica adecuada en el grupo $100 \mathrm{\mu g}$ iniciales frente al grupo 200 $\mu g$ iniciales de CBF. La dosis inicial de $100 \mathrm{\mu g}$ de CBF no se mostró estadísticamente inferior a la de $200 \mathrm{\mu g}^{(25)}$. La valoración de la respuesta se registró según el criterio del paciente.

\begin{tabular}{|l|c|c|}
\cline { 2 - 3 } \multicolumn{1}{c|}{} & $\begin{array}{c}\text { Grupo 100 } \mu g \\
(\mathrm{n}=145)\end{array}$ & $\begin{array}{c}\text { Grupo 200 } \mu \mathrm{g} \\
(\mathrm{n}=167)\end{array}$ \\
\hline \% de pacientes con respuesta analgésica adecuada & 75,2 & 81,4 \\
\hline \% diferencia entre los grupos & \multicolumn{2}{|c|}{$-6,3$} \\
\hline \% límite superior del IC & \multicolumn{2}{|c|}{1,4} \\
\hline
\end{tabular}


cimientos adversos inaceptables con una dosis inicial de $100 \mu \mathrm{g}$ de CBF frente a una dosis inicial de $200 \mu \mathrm{g}$. Los resultados se exponen en la tabla 7 y los autores concluyeron que la dosis inicial de $100 \mu \mathrm{g}$ de CBF no es inferior a la $200 \mu \mathrm{g}$. La dosis adecuada que se indicó con más frecuencia fue $200 \mu \mathrm{g}(39,6 \%)$ y $400 \mu \mathrm{g}(26,9 \%)$.

El $6,7 \%$ de los pacientes notificaron efectos adversos relacionados con el tratamiento, cada uno de ellos notificados por un paciente, excepto dos pacientes con náusea y 4 con edema en el lugar de aplicación.

\section{DISCUSIÓN}

El tratamiento del DIO requiere un fármaco de potencia adecuada, inicio de acción rápido y corta duración. La evidencia disponible muestra que el CBF es eficaz en términos de alivio del dolor en el tratamiento de los episodios de DIO.

El inicio de acción del CBF es muy rápido, unos 10 minutos, y comparando con fentanilo bucal no efervescente o citrato de fentanilo oral transmucoso, se observa que la absorción del CBF fue más rápida ( $\mathrm{T}_{\max }$ más corta) y más completa (mayor AUC) y los niveles séricos mayores. La velocidad del inicio de acción es un aspecto relevante en el tratamiento de estos pacientes, aunque en la actualidad solo un 9\% de los episodios de DIO se tratan con opioides de acción rápida ${ }^{(27)}$.

El perfil de absorción y los efectos adversos observados en pacientes con mucositis de intensidad clínica 1-3 son comparables a los pacientes sin mucositis sugiriendo que no es necesario el ajuste de dosis en estas condiciones. Cabe destacar que no se dispone de datos en pacientes con mucositis clínica con grado superior a 3 .

En pacientes que requieren un cuidador para la administración del fármaco, xerostomía severa o determinadas condiciones de salud bucodental, el uso del CBF vía sublingual es una alternativa viable ya que la ratio bucal/sublingual de la medias de la Cmax y el AUC $\infty$ se mostraron dentro del rango de bioequivalencia.

En general el tratamiento con CBF se muestra bien tolerado y tiene un perfil de seguridad favorable. La mayoría de los acontecimientos adversos registrados fueron leves o moderados y los graves se relacionaron principalmente con la enfermedad oncológica de base. El tratamiento con opioides de acción prolongada utilizados para tratar el dolor basal debe estabilizarse antes de añadir CBF y el paciente debe proseguir el tratamiento con el opioide de larga duración mientras se administra el CBF, siendo también aconsejable monitorizar al paciente durante el proceso de ajuste de dosis y considerar el riesgo potencial de depresión respiratoria.

ACRONIMOS: AUC: área bajo la curva. Indica la concentración o absorción de un medicamento en función de un período de tiempo. Cmax: concentración plasmática máxima. CBF: comprimido bucal de fentanilo. CFOT: citrato de fentanilo oral transmucoso. IC: Intervalo de confianza. OD: odds ratio. PI: intensidad del dolor. SPID: sumatoria de la diferencia en la intensidad del dolor. Tmax: tiempo necesario para alcanzar la concentración máxima de un fármaco

\section{REFERENCIAS BIBLIOGRÁFICAS}

1. Portenoy RK, Hagen NA. Breakthrough pain: definition, prevalence and characteristics. Pain 1990 41:273-81.

2. Zeppetella G, O'Doherty CA, Collins $\mathrm{S}$. Prevalence and characteristics of breakthrough pain in cancer patients admitted to a hospice. J Pain Symptom Manage 2000;20:87-92. Doi: 10.1016/ S0885-3924(00)00161-5 
3. Portenoy RK, Taylor D, Messina J, Tremmel L. A randomized, placebo-controlled study of fentanyl buccal tablet for breakthrough pain in opioid-treated patients with cancer. Clin J Pain 2006;22:805-11. Doi: 10.1097/01.ajp.0000210932.27945.4a

4. Slatkin NE, Xie F, Messina J, Segal TJ. Fentanyl buccal tablet for relief of breakthrough pain in opioid-tolerant patients with cancer-related chronic pain. J Support Oncol 2007; 5:327-34

5. Zeppetella G, Messina J, Xie F, Slatkin NE. Consistent and clinically relevant effects with fentanyl buccal tablet in the treatment of patients receiving maintenance opioid therapy and experiencing cancerrelated breakthrough pain. Pain Practice 2010;10:287-93. Doi: 10.1111/j.15332500.2010.00359.x

6. Pather SI, Siebert JM, Hontz J, Khankari RK, Gupte SV, Kumbale R. Enhanced buccal delivery of fentanyl using the OraVescent drug delivery system. Drug Deliv Tech 2001;1:54-7

7. Darwish $M$, Kirby $M$, Robertson $P$ Jr, Tracewell W, Jiang JG. Absolute and relative bioavailability of fentanyl buccal tablet and oral transmucosal fentanyl citrate. J Clin Pharmacol 2007;47:343-50 Doi: 10.1177/0091270006297749

8. Darwish $M$, Kirby $M$, Robertson $P$ Jr, Tracewell W, Xie F. Dose proportionality of fentanyl buccal tablet in doses ranging from 600 to 1300 microg in healthy adult subjects: A randomized, open-label, four-period, crossover, single-centre study. Clin Drug Investig 2010;30:365-73. Doi: 10.2165/11533360-000000000-00000.

9. Darwish M, Kirby M, Jiang JG, Tracewell W, Robertson P. Following buccal and sublingual placement of Fentanyl buccal tablet $400 \mu \mathrm{g}$ in healthy subjects bioequivalence following buccal and sublingual placement of Fentanyl buccal tablet $400 \mu \mathrm{g}$ in healthy subjects. Clin Drug Invest 2008;28: 1-7.

10. Darwish M, Kirby M, Robertson P, Trace- well W, Jiang JG. Absorption of fentanyl from fentanyl buccal tablet in cancer patients with or without oral mucositis. Clin Drug Invest 2007;27:605-11. Doi: 10.2165/00044011-200727090-00002

11. Weinstein S, Messina J, Xie F. Fentanyl buccal tablet for the treatment of breakthrough pain in opioid-tolerant patients with chronic cancer pain. Cancer 2009;115:2571-9. Doi: 10.1002/cncr.24279.

12. Mercadante S, Gatti A, Porzio G, Lo Presti C, Aielli F, Adile C, Casuccio A. Dosing fentanyl buccal tablet for breakthrough cancer pain: Dose titration versus proportional doses. Curr Med Res Opin 28(6):963-8. Doi: 10.1185/03007995.2012.683112

13. Finn AL, Vasisht N, Stark JG, Gever LN, Tagarro I. Dose proportionality and pharmacokinetics of fentanyl buccal soluble film in healthy subjects: A phase I, open-label, three-period, crossover study. Clin Drug Investig. 2012;32:63-71. Doi: 10.2165/11594670-000000000-00000.

14. Ashburn MA, Slevin KA, Messina J, Xie F. The efficacy and safety of fentanyl buccal tablet compared with immediaterelease oxycodone for the management of breakthrough pain in opioid-tolerant patients with chronic pain. Anesth Analg 2011; 112(3):693-702. Doi: 10.1213/ ANE.0b013e318209d320.

15. Mercadante S, Ferrera P, Arcuri E. The use of fentanyl buccal tablets as breakthrough medication in patients receiving chronic methadone therapy: An open label preliminary study. Support Care Cancer 2011;19:435-8. Doi: 10.1007/s00520010-1015-6

16. Farrar JT, Messina J, Xie F, Portenoy RK.A novel 12-week study, with three randomized, double-blind placebo-controlled periods to evaluate fentanyl buccal tablets for the relief of breakthrough pain in opioidtolerant patients with noncancer-related chronic pain. Pain Med 2010;11:1313-27. Doi: 10.1111/j.1526-4637.2010.00939.x.

17. Fine PG, Messina J, Xie F, Rathmell J. 
Long-term safety and tolerability of fentanyl buccal tablet for the treatment of breakthrough pain in opioid-tolerant patients with chronic pain: An 18-month study. J Pain Symptom Manage 2010;40:747-60. Doi: 10.1016/j.jpainsymman.2010.02.009

18. Vasisht N, Gever LN, Tagarro I, Finn AL. Evaluation of the single- and multipledose pharmacokinetics of fentanyl buccal soluble film in normal healthy volunteers. J Clin Pharmacol 2010;50:785-91. Doi: 10.1177/0091270010361354.

19. Rauck R, North J, Gever LN, Tagarro I. Fentanyl buccal soluble film (FBSF) for breakthrough pain in patients with cancer: A randomized, double-blind, placebo-controlled study. Ann Oncol 2010;21:130814. Doi: 10.1093/annonc/mdp541

20. Vasisht N, Gever LN, Tagarro I, Finn AL. Formulation selection and pharmacokinetic comparison of fentanyl buccal soluble film with oral transmucosal fentanyl citrate: A randomized, open-label, singledose, crossover study. Clin Drug Investig 2009;29:647-54. doi: 10.2165/11315300000000000-00000.

21. Simpson DM, Messina J, Xie F, Hale $M$. Fentanyl buccal tablet for the relief of breakthrough pain in opioid-tolerant adult patients with chronic neuropathic pain: A multicenter, randomized, double-blind, placebo-controlled study. Clin Ther 2007;29:588-601. Doi10.1016/j. clinthera.2007.04.007

22. Hanks GW, Nugent M, Higgs CM, Busch MA; OTFC Multicentre Study Group.. Oral transmucosal fentanyl citrate in the management of breakthrough pain in cancer: An open, multicentre, dose-titration and long-term use study. Palliat Med
2004;18:698-704.

23. Christie JM, Simmonds M, Patt R, Coluzzi P, Busch MA, Nordbrock E, et al. Dosetitration, multicenter study of oral transmucosal fentanyl citrate for the treatment of breakthrough pain in cancer patients using transdermal fentanyl for persistent pain. J Clin Oncol 1998; 16:3238-45.

24. Darwish M, Tempero K, Kirby M, Thompson J. Relative bioavailability of the fentanyl effervescent buccal tablet (FEBT) 1,080 pg versus oral transmucosal fentanyl citrate 1,600 pg and dose proportionality of FEBT 270 to 1,300 microg: A single-dose, randomized, open-label, three-period study in healthy adult volunteers. Clin Ther 2006; 28:715-24. Doi: 10.1016/j.clinthera.2006.05.016

25. Davies A, Jarosz J, Kleeberg UR, Mercadante S, O'Brien T, Poulain P, et al. Pan-European Phase IV Open-Label Multicentre Study in Patients With Breakthrough Cancer Pain (BTcP) Treated With Fentanyl Buccal Tablet (FBT): Titration. Poster presented at 7th World Research Congress of the European Association for Palliative Care. Trondheim. June 2012.

26. Davies A, Jarosz J, Kleeberg UR, Mercadante S, O'Brien T, Poulain P, et al. Pan-European Phase IV Open-Label Multicentre Study in Patients with Breakthrough Cancer Pain (BTcP) treated with Fentanyl buccal tablet: Quality of Life. Poster presented at 7th World Research Congress of the European Association for Palliative Care. Trondheim. June 2012.

27. Gomez-Batiste X, Madrid F, Moreno F, Gracia A, Trelis J, Nabal M, et al. Breakthrough cancer pain: Prevalence and characteristics in patients in Catalonia, Spain. J Pain Symptom Manage 2002;24:45-52. Doi: 10.1016/S0885-3924(02)00421-9. 
